\title{
Abordagem dos cirurgiões-dentistas do Estratégia de Saúde da Família do município de Patos-PB a usuários com hipertensão arterial
}

\author{
Approach of dentists of the family health strategy of the city of Patos-PB \\ to users with hypertension
}

Izabelle Maria Ferreira da Silva Vale*

Rosana Araújo Rosendo*

Luciana Ferraz Gominho***

Michele Baffi Diniz ${ }^{* * *}$

Dmitry José de Santana Sarmento ${ }^{* * * *}$

Tássia Cristina de Almeida Pinto-Sarmento ${ }^{* * * *}$

\section{Resumo}

Objetivo: o objetivo deste trabalho é verificar o conhecimento dos cirurgiões-dentistas dos Estratégias de Saúde da Família (ESF) do município de Patos-PB, no atendimento a usuários com hipertensão arterial (HA). Materiais e métodos: é um estudo do tipo transversal e analítico, no qual foi aplicado um questionário estruturado com 21 profissionais dos ESFs de Patos-PB. Os dados obtidos foram analisados a partir da estatística descritiva e inferencial bivariada (testes Qui-Quadrado e Exato de Ficher). Resultados: dos pesquisados, 85,7\% definiram hipertensão arterial, mas apenas 44,4\% desses responderam corretamente; 95,2\% responderam corretamente o valor normal da pressão arterial. A xerostomia foi a alteração causada pelo uso de anti-hipertensivo mais citada (85,71\%); a lidocaína foi o anestésico de escolha (61,9\%); a interação medicamentosa mais citada $(48,6 \%)$ foi a dos anti-hipertensivos com os anti-inflamatórios não estereoidais e com os analgésicos com ácido acetilsalicílico (AAS). Participam do Programa Hiperdia $66,7 \%$ dos entrevistados e $33,3 \%$ relataram aferir a pressão dos usuários, dependendo da anamnese realizada. Observou-se a associação estatística entre trabalhar em outro local e definir correta- mente a HA $(p=0,006)$. Conclusão: os profissionais que participaram da pesquisa mostraram um conhecimento relativamente adequado/producente, realizando uma conduta condizente com o que a literatura sugere, mas, ainda, necessitando de maiores esclarecimentos sobre o tema, para suprir suas dificuldades.

Palavras-chave: Anestesia local. Hipertensão. Odontologia. Vasoconstritores.

\section{Introdução}

Define-se hipertensão arterial (HA) como uma cardiopatia caracterizada pelo aumento anormal da pressão arterial. Se não houver o controle adequado desses pacientes, sérias complicações poderão aparecer, como acidentes cerebrovasculares, problemas renais e trombose, entre outros, tornando-se um importante agravo à saúde pública não somente no Brasil, mas também no mundo ${ }^{1}$.

Cirurgiã-dentista, graduada pela Universidade Federal de Campina Grande (UFCG), Patos, Paraíba, PB, Brasil .

Mestra em Diagnóstico Bucal; professora da Faculdade de Odontologia da Universidade Federal de Campina Grande (UFCG), Patos, Paraíba, PB, Brasil.

Doutor em Endodontia; professora da Faculdade de Odontologia da Universidade Federal de Campina Grande (UFCG), Patos, Paraíba, PB, Brasil. Doutor em Ciências Odontológicas; professora da Faculdade de Odontologia da Universidade Cruzeiro do Sul, São Paulo, SP, Brasil.

Mestre em Patologia Oral; professor da Universidade Estadual da Paraíba (UEPB), Araruna, Paraíba, PB, Brasil.

* Mestra em Clínica Odontológica; professora da Faculdade de Odontologia da Universidade Federal de Campina Grande (UFCG), Patos, Paraíba, PB, Brasil. 
O quadro de HA pode ser definido quando a pressão arterial sistólica (PAS) for maior ou igual a 140 $\mathrm{mmHg}$ e quando a pressão arterial diastólica (PAD) for maior ou igual a $90 \mathrm{mmHg}$ em indivíduos que não façam uso de medicação anti-hipertensiva, em mais de uma ocasião ${ }^{2}$.

Quanto à epidemiologia, dados mostram que aproximadamente 30 milhões de brasileiros são hipertensos, mas que metade dessas pessoas não sabem da existência do problema ${ }^{1}$. Segundo a sua etiologia, pode ser classificada em primária, quando não há outra patologia associada que explique o aumento da pressão arterial (PA), ou em secundária quando se encontra associada a outra patologia que cause a elevação da PA, nesse caso, passível de cura mediante a eliminação da causa ${ }^{3}$.

Para os cirurgiões-dentistas é muito importante conhecer as possíveis complicações que podem acontecer durante o atendimento odontológico ao paciente hipertenso, assim como também as consequências provocadas devido à medicação utilizada por esses. Os anti-hipertensivos são fármacos que podem provocar algumas alterações bucais, como a hiperplasia do tecido gengival e a diminuição do fluxo salivar ${ }^{4}$.

Outro fator que merece destaque é a problemática da utilização de anestésicos locais com vasoconstritores em pacientes hipertensos, pois se observa que há o receio dos cirurgiões-dentistas de realizarem essa conduta, visto que se aplicada de forma incorreta possa causar o agravamento da doença ${ }^{1}$.

Problemas relacionados a interações medicamentosas podem influenciar de forma negativa na saúde do paciente, provenientes do aparecimento de efeitos adversos ou da falha no objetivo terapêutico ${ }^{5}$. Assim, todos os anti-inflamatórios não estereoidais (Aines) podem realizar uma ação contrária de forma parcial ou total sobre os efeitos de vários agentes anti-hipertensivos, aumentando a morbidade desses pacientes, podendo levar a crises hipertensivas ${ }^{6}$.

É muito importante ressaltar a necessidade do cirurgião-dentista ter conhecimento específico sobre as particularidades desses pacientes, no intuito de poder oferecer um atendimento mais seguro e eficaz, sem correr o risco de causar complicações não apenas locais, mas também sistêmicas ${ }^{7}$.

Diante do exposto, o presente estudo avalia a percepção dos cirurgiões-dentistas do Estratégia de Saúde da Família em relação à abordagem a usuários com hipertensão arterial, quais as condutas adotadas na realização do tratamento desses, averiguando as principais dificuldades encontradas pelos profissionais.

\section{Materiais e métodos}

Foi realizada uma pesquisa com 21 cirurgiões-dentistas dos Estratégias de Saúde da Família do município de Patos, no estado da Paraíba. A coleta dos dados foi realizada por meio de um questionário estruturado com 21 perguntas, o qual foi adaptado de estudo anteriormente realizado ${ }^{8}$.

De acordo com a resolução 466/12 do Conselho Nacional de Saúde (CNS), esse trabalho foi aprovado pelo Comitê de Ética em Pesquisa (CAAE 35706714.0.0000.5181) e segue os padrões exigidos pela Declaração de Helsink, e previamente a coleta dos dados foi explicado ao participante com o objetivo da pesquisa, sendo solicitada à sua participação por meio da assinatura do Termo de Consentimento Livre e Esclarecido. A aplicação dos questionários foi feita nos intervalos entre $o$ atendimento de um usuário e outro, no próprio consultório da unidade. A autenticidade das respostas foi avaliada pelo método de "face", em que 10\% dos entrevistados explicaram as perguntas com suas próprias palavras, o que permite concluir que o instrumento foi bem compreendido pelos participantes ${ }^{9}$.

$\mathrm{O}$ instrumento de pesquisa contemplou questões referentes à formação do profissional, informações sobre a definição da HA, o valor da PA normal, o hábito de verificar a PA dos usuários, as manifestações bucais presentes nos usuários que fazem uso crônico de medicamentos anti-hipertensivos, bem como questões relacionadas ao uso de anestésicos e fármacos de utilização sistêmica.

A definição de PA foi avaliada segundo a definição da Sociedade Brasileira de Hipertensão (SBH) que considera a patologia como uma elevação crônica da PA sistólica ou diastólica ${ }^{10}$, sendo considera satisfatória aquela resposta totalmente correta, insatisfatória quando estava errada e incompleta quando faltava alguma informação na descrição.

Utilizou-se como critério de inclusão os cirurgiões-dentistas atuantes nas unidades básicas de saúde da rede pública do município, presentes nessas unidades nos dias da aplicação dos questionários, que concordaram em participar da pesquisa, assinando o Termo de Consentimento Livre e Esclarecido (TCLE). Foram excluídos da pesquisa os profissionais que se negaram em responder ao questionário e os que se encontravam em seu período de férias, além daqueles que atuassem nas unidades que se encontrassem fechadas.

Os dados obtidos neste estudo foram tabulados no programa Statistical Package for Social Sciences (SPSS) versão 18.0 e foram apresentados na forma de gráficos, com apresentação de valores absolutos e percentuais. Para análise estatística dos dados foi utilizado o teste do Qui-Quadrado e Exato de Fischer com nível de significância de 5\%.

\section{Resultados}

No tocante à caracterização do perfil da amostra, observou-se que dos cirurgiões-dentistas entrevistados, $61,9 \%$ dos profissionais eram do sexo feminino, de faixa etária entre 22 e 61 anos, com média de 33,86 ( $\pm 12,39)$ (Tabela 1). A maior parte 
desses trabalhava em outro emprego $(69,1 \%)$, sendo que $68,8 \%$ eram em consultório particular e apenas $6,2 \%$ atuavam em clínica com outros profissionais. A maioria dos entrevistados estavam formados há menos de cinco anos (61,9\%), 14,3\% tinham entre seis e dez anos de profissão e $23,8 \%$ tinham mais de dez anos de formação, variando esse tempo de dois meses a 33 anos, com média de 9,6 anos e desvio padrão de 11,66.

Verifica-se ainda que $71,4 \%$ estão no emprego há apenas cinco anos ou menos e que $36,4 \%$ não têm qualquer pós-graduação, mas dos que têm $59,1 \%$ são especialistas e 4,5\% têm mestrado, lembrando que os participantes podiam responder a mais de uma alternativa. Dentre as especialidades encontradas, Saúde da Família foi a mais citada, representando $61,5 \%$ dos dados. Quando perguntados se participavam do Programa Hiperdia, 66,7\% responderam que sim (Tabela 1 ).

Tabela 1 - Caracterização da amostra

\begin{tabular}{l|r|r}
\hline \multicolumn{1}{|c|}{ Variáveis } & $\mathrm{N}$ & $\%$ \\
\hline Sexo & & \\
Masculino & 8 & 38,1 \\
Feminino & 13 & 61,9 \\
Trabalha em outro lugar & & \\
Sim & 13 & 61,9 \\
Não & 8 & 38,1 \\
Qual outro emprego* & & \\
Consultório particular & 11 & 68,8 \\
Clínica & 1 & 6,2 \\
Faculdade & 2 & 12,5 \\
Outro serviço público & 2 & 12,5 \\
Tempo de formado & & \\
< 5 anos & 13 & 61,9 \\
6-10 anos & 3 & 14,3 \\
> 10 anos & 5 & 23,8 \\
Tempo que está neste emprego & & \\
Até 5 anos & 15 & 71,4 \\
Mais de 5 anos & 6 & 28,6 \\
Tem pós-graduação* & 13 & 66,7 \\
Não & 7 & 33,3 \\
Especialização & 1 & 36,4 \\
Mestrado & 1 & 7,1 \\
Endodontia & 2 & 15,4 \\
Pacientes com necessidades especiais & 1 & 7,7 \\
Ortodontia & 8 & 61,5 \\
Prótese & & \\
Saúde da famólia & 14 \\
\hline
\end{tabular}

Fonte: dos autores.

- Os participantes poderiam responder mais de uma alternativa.
Pode-se observar o conhecimento dos cirurgiões-dentistas acerca da hipertensão arterial, constatando-se que 85,7\% afirmaram saber definir HA, mas apenas $44,4 \%$ responderam de maneira satisfatória, de modo semelhante $44,4 \%$ responderam insatisfatoriamente e $11,2 \%$ forneceram uma resposta incompleta. Acerca dos tipos de HA, 52,4\% da amostra afirmou não saber quais eram, e dos que afirmavam conhecer, $50 \%$ responderam corretamente quais são esses tipos. Acrescenta-se a esses dados que $95,2 \%$ souberam responder corretamente o valor da pressão arterial normal (Tabela 2).

Tabela 2 -Conhecimento dos cirurgiões-dentistas acerca da hipertensão arterial

\begin{tabular}{l|r|r}
\hline \multicolumn{1}{c|}{ Variáveis } & $\mathrm{N}$ & $\%$ \\
\hline Sabe definir HA? & 18 & 85,7 \\
Sim & 3 & 14,3 \\
Não & & \\
Qualidade de definição & 8 & 44,4 \\
Satisfatória & 8 & 44,4 \\
Insatisfatória & 2 & 11,2 \\
Incompleta & & \\
Conhece os tipos de HA? & 10 & 47,6 \\
Sim & 11 & 52,4 \\
Não & & \\
Conhece corretamente os tipos de HA & 5 & 50,0 \\
Sim & 5 & 50,0 \\
Não & 20 & 95,2 \\
Respondeu corretamente o valor da PA normal & 1 & 4,8 \\
$\quad$ Sim &
\end{tabular}

Na Tabela 3, pode-se constatar que dentre as manifestações bucais causadas pelo uso de anti-hipertensivos, a xerostomia foi a mais citada, obtendo $85,7 \%$ das respostas, e a glossite foi citada em apenas $9,5 \%$ das respostas. Quando indagados sobre as soluções anestésicas que podem ser utilizadas nos usuários hipertensos, 61,9\% responderam lidocaína e 9,5\% responderam articaína e igual número bupivacaína. Sobre como minimizar a ansiedade do paciente, $95,8 \%$ afirmaram conversar com os pacientes antes dos procedimentos. As interações medicamentosas entre os Aines e os anti-hipertensivos que o paciente possa estar utilizando, mostraram-se conhecidas por apenas 48,6\%. Por último, quanto à conduta desses profissionais, quando perguntados em que casos aferiam a PA do usuário, $33,3 \%$ responderam que dependia da anamnese realizada e $4,8 \%$ não souberam responder. 
Tabela 3 - Prática clínica dos cirurgiões-dentistas com o usuário hipertenso

\begin{tabular}{|c|c|c|}
\hline Variáveis & $\mathrm{N}$ & $\%$ \\
\hline \multicolumn{3}{|l|}{ Manifestações bucais mais frequentes* } \\
\hline Xerostomia & 18 & 85,7 \\
\hline Reações liquenoides & 4 & 19,0 \\
\hline Úlceras & 4 & 19,0 \\
\hline Alteração do paladar & 12 & 57,1 \\
\hline Crescimento gengival & 8 & 38,0 \\
\hline Sensação de gosto metálico & 6 & 28,5 \\
\hline Glossite & 2 & 9,5 \\
\hline Angioedema & 3 & 14,2 \\
\hline \multicolumn{3}{|c|}{ Soluções anestésicas que podem ser utilizadas* } \\
\hline Lidocaína & 13 & 61,9 \\
\hline Citocaína & 9 & 42,8 \\
\hline Mepivacaína & 11 & 52,4 \\
\hline Prilocaína & 9 & 48,6 \\
\hline Articaína & 2 & 9,5 \\
\hline Bupivacaína & 2 & 9,5 \\
\hline \multicolumn{3}{|c|}{ Como minimiza situações de ansiedade* } \\
\hline Fármacos benzodiazepínicos & 1 & 4,8 \\
\hline Conversas com o usuário & 20 & 95,2 \\
\hline Outros & 2 & 9,5 \\
\hline \multicolumn{3}{|l|}{ Interação medicamentosa* } \\
\hline Penicilinas & 3 & 14,3 \\
\hline Aines & 9 & 48,6 \\
\hline Analgésicos & 1 & 4,8 \\
\hline Cefalosporinas & 1 & 4,8 \\
\hline Analgésicos com AAS & 9 & 48,6 \\
\hline Macrolídeos & 3 & 14,2 \\
\hline Anti-inflamatórios estereoidais & 7 & 33,3 \\
\hline \multicolumn{3}{|l|}{ Interação com outros profissionais } \\
\hline Sim & 19 & 90,5 \\
\hline Não & 2 & 9,5 \\
\hline \multicolumn{3}{|c|}{$\begin{array}{l}\text { Quantidade de tubetes de anestésico utilizada } \\
\text { no usuário hipertenso }\end{array}$} \\
\hline Até dois tubetes & 9 & 42,9 \\
\hline Mais de dois tubetes & 11 & 52,4 \\
\hline Não respondeu & 1 & 4,7 \\
\hline \multicolumn{3}{|l|}{ Quando afere a PA do usuário } \\
\hline Usuários com menos de 40 anos & 4 & 19,0 \\
\hline Usuários com 40 anos ou mais & 6 & 28,6 \\
\hline Depende da anamnese & 7 & 33,3 \\
\hline Em procedimentos cirúrgicos & 1 & 4,8 \\
\hline Não afere & 2 & 9,5 \\
\hline Não respondeu & 1 & 4,8 \\
\hline
\end{tabular}

Fonte: dos autores.

* Os participantes podiam responder mais de uma alternativa.
Ao se realizar a avaliação do perfil da amostra em relação à correta definição de hipertensão arterial, observou-se a associação estatística $(\mathrm{p}=0,006)$ entre os cirurgiões-dentistas que trabalham em outros locais além da Estratégias de Saúde da Família (ESFs) e o conhecimento da correta definição de hipertensão arterial (Tabela 4).

Tabela 4 - Relação entre a categorização da amostra e a correta definição de hipertensão arterial

\begin{tabular}{|c|c|c|c|c|}
\hline \multirow[b]{2}{*}{ Variáveis } & \multicolumn{4}{|c|}{ Correta definição } \\
\hline & $\begin{array}{l}\text { Sim } \\
\mathrm{n}(\%)\end{array}$ & $\begin{array}{c}\text { Não } \\
\mathrm{n}(\%) \\
\end{array}$ & $\begin{array}{c}\text { Total } \\
\mathrm{n}(\%)\end{array}$ & $\begin{array}{c}\text { Valor de } \\
\qquad \mathrm{p}^{(1)}\end{array}$ \\
\hline \multicolumn{5}{|c|}{$\begin{array}{l}\text { Tempo que está neste } \\
\text { emprego }\end{array}$} \\
\hline Até 5 anos & $7(46,7)$ & $8(53,3)$ & $15(100,0)$ & \multirow{2}{*}{0,221} \\
\hline Mais de 5 anos & $1(16,7)$ & $5(83,3)$ & $6(100,0)$ & \\
\hline \multicolumn{5}{|c|}{ Trabalha em outro local } \\
\hline Não & $0(0,0)$ & $8(100,0)$ & $8(100,0)$ & \multirow{2}{*}{0,006} \\
\hline Sim & $8(61,5)$ & $5(38,5)$ & $13(100,0)$ & \\
\hline \multicolumn{5}{|l|}{ Tempo de formado } \\
\hline$<5$ anos & $6(46,2)$ & $7(53,8)$ & $13(100,0)$ & \multirow{3}{*}{$0,582^{(2}$} \\
\hline 6-10 anos & $1(33,3)$ & $2(66,7)$ & $3(100,0)$ & \\
\hline$>10$ anos & $1(20,0)$ & $4(80,0)$ & $5(100,0)$ & \\
\hline \multicolumn{5}{|l|}{ Tem pós-graduação } \\
\hline Não & $4(50,0)$ & $4(50,0)$ & $8(100,0)$ & \multirow{2}{*}{0,336} \\
\hline Sim & $4(30,8)$ & $9(69,2)$ & $13(100,0)$ & \\
\hline \multicolumn{5}{|l|}{ Participa do hiperdia } \\
\hline Não & $1(14,3)$ & $6(85,7)$ & $7(100,0)$ & \multirow{2}{*}{0,133} \\
\hline Sim & $7(50,0)$ & $7(50,0)$ & $14(100,0)$ & \\
\hline
\end{tabular}

Fonte: dos autores.

(1) teste Exato de Fischer / *Significante ao nível de 5\%.

(2) teste Qui-Quadrado.

\section{Discussão}

No Brasil, existem cerca de 17 milhões de hipertensos, dos quais $35 \%$ encontram-se numa faixa etária acima dos 40 anos de idade. Sendo assim, estratégias de saúde têm sido criadas desde a prevenção ao tratamento desses usuários. O Estratégia de Saúde da Família é o principal programa responsável pela atenção básica, atuando com uma equipe multiprofissional, em que o processo de trabalho desenvolve um elo com a comunidade ${ }^{11}$.

Nesse contexto, dos 38 cirurgiões-dentistas pertencentes ao Estratégia Saúde da Família do município de Patos-PB, foram entrevistados 21. A maior parte apresentava pós-graduação, o que demonstrou o interesse desses profissionais por atualizações. Nove profissionais não foram encontrados após duas tentativas por estarem em férias, sete negaram-se a responder e uma unidade encontrava-se fechada.

As principais dificuldades, na realização deste trabalho, foram a relativa falta de interesse por parte dos profissionais em responder o questionário, bem como a falta de estudos com essas características, que dificultaram a confrontação dos dados. Dessa forma, foi realizado um confronto entre as respostas dos sujeitos com as encontradas na literatura para a realização das análises dos resultados. 
Estudos na literatura ${ }^{12,13}$ concordam quando afirmam que a hipertensão é a doença sistêmica mais frequente nos consultórios odontológicos. Esses dizem ainda que essa enfermidade pode ser diagnosticada precocemente pela aferição casual da PA. Mesmo assim, no estudo realizado constatou-se que apenas $33,3 \%$ dos profissionais realizavam a aferição da pressão arterial de acordo com as necessidades encontradas na anamnese, encontrando resultado semelhante em estudo realizado na zona leste de São Paulo-SP ${ }^{14}$, no qual $75 \%$ dos cirurgiões-dentistas habitualmente não aferem a PA.

Em trabalho, avaliando o aumento da PA dos pacientes durante o atendimento odontológico ${ }^{15}$, foi observado que aqueles que apresentavam uma característica de ansiedade elevada, apresentavam aumento na PAS em alguns procedimentos, sugerindo que esse aumento poderia estar associado ao medo ou à ansiedade. Desse modo, para minimizar essas crises de ansiedade, evitando a elevação da PA, existem métodos farmacológicos como o uso de benzodiazepínicos, e os não farmacológicos que utilizam a conversa associada ou não a um relaxamento muscular ${ }^{16}$. Diante disso, no questionário foi elaborada uma pergunta aos profissionais para saber como minimizar a ansiedade nos usuários, visto que de maneira concordante com o referido autor, $95,8 \%$ dos entrevistados responderam que a conversa antes dos procedimentos era a melhor maneira de controlar a ansiedade nos usuários.

A pressão arterial pode ser classificada em quatro categorias de acordo com a Joint National Committee: normal com a PAS < $120 \mathrm{mmHg}$ e PAD $<80$ $\mathrm{mmHg}$; pré-hipertenso com a PAS entre 120-139 $\mathrm{mmHg}$ e a PAD entre $80-89 \mathrm{mmHg}$; estágio $1 \mathrm{com}$ a PAS entre $140-159 \mathrm{mmHg}$ e a PAD entre $90-99$ $\mathrm{mmHg}$, e o estágio 2 com a $\mathrm{PAS} \geq 160 \mathrm{mmHg}$ e $\mathrm{PAD} \geq$ $100 \mathrm{mmHg}^{4,17,18}$. Assim, de maneira bastante positiva este estudo constatou que 95,2\% souberam responder corretamente o valor da pressão arterial normal. Desse modo, observa-se que a maior parte dos profissionais sabe diferenciar a condição de normalidade para uma alteração patológica, sendo possível a detecção de um provável quadro de hipertensão.

A ocorrência de alterações no sistema estomatognático de pacientes hipertensos é causada principalmente pela utilização crônica das medicações anti-hipertensivas ${ }^{19,20}$. Nesse contexto, é possível observar na literatura que o efeito colateral de maior relevância encontrado nos pacientes hipertensos é a xerostomia ${ }^{21-23}$. De forma semelhante, a xerostomia, nesta pesquisa, foi a mais citada em $85,71 \%$ das respostas como a principal manifestação bucal devido ao uso dos anti-hipertensivos.

Ainda se torna relevante abordar, nesta temática, a importância do cuidado na utilização de anestésicos locais nos pacientes hipertensos, sendo essa interação ressaltada em estudo sobre a interação do uso da adrenalina na anestesia local odontológica em portador de coronariopatia ${ }^{24}$. Nesse contexto de utilização das anestesias locais, constata-se que, em pacientes hipertensos compensados, o uso de vasoconstritores adicionados às soluções anestésicas locais não é contraindicado, de modo que pode ser utilizada a adrenalina 1:100.000 ou 1:200.000, em doses pequenas, e o mais indicado é não ultrapassar o limite de dois tubetes por sessão. Outra opção existente é o uso da felipressina $0,03 \mathrm{UI} / \mathrm{ml}$ como vasoconstritor, associado à prilocaína $3 \%$, por não produzir efeitos no sistema cardiovascular ${ }^{25,26}$. Entretanto, é possível ainda observar, na literatura, a indicação da mepivacaína a $3 \%$ sem vasoconstrictor como anestésico mais seguro a se utilizar em pacientes hipertensos descompensados ${ }^{27}$.

Diante de tudo que já foi apresentado, muitos são os fatores que influenciam na melhor conduta a ser seguida, pois fatores como o tipo de hipertensão, o quadro emocional do paciente, o procedimento a ser realizado e o tempo do procedimento, entre outros, influenciam bastante na tomada de decisão do cirurgião-dentista durante o seu atendimento e qual a conduta a ser realizada, tendo suas exceções àquilo que é preconizado.

Seguindo esse mesmo contexto, perguntou-se, neste estudo, aos profissionais a respeito da quantidade e do tipo de anestésico utilizado nos pacientes hipertensos. Em relação à quantidade de tubetes, os profissionais obtiveram um percentual de erro de $52,4 \%$, mas houve um comportamento diferente nas respostas fornecidas em relação ao tipo de anestésico usado em pacientes hipertensos, tendo um maior percentual de acerto em $61,9 \%$.

Grande parte da literatura pesquisada concorda que a realização do tratamento em pacientes hipertensos se dá por meio da utilização de vários fármacos denominados de anti-hipertensivos, que são: diuréticos, inibidores da enzima conversora de angiotensina-IECA, beta-bloqueadores, inibidores adrenérgicos, vasodilatadores e bloqueadores dos canais de cálcio ${ }^{6,28,29}$, sendo de fundamental importância que os diversos profissionais da saúde tenham conhecimento sobre esses fármacos, a fim de minimizar o risco das associações medicamentosas prejudiciais ${ }^{30}$.

Diante dessa temática, confere-se que todos os Aines podem realizar uma ação contrária de forma parcial ou total sobre os efeitos de vários agentes anti-hipertensivos, aumentando a morbidade desses pacientes, podendo levar a crises hipertensivas ${ }^{6}$. De maneira preocupante, os dados obtidos nesta pesquisa mostraram que apenas $48,6 \%$ dos entrevistados conhecem as interações desses medicamentos. Dessa forma, dentre os medicamentos que causam interações medicamentosas prejudiciais, os Aines são os mais importantes para os cirurgiões-dentistas, uma vez que essa classe de medicamentos são as mais prescritas por esses profissionais, cabendo a eles conhecerem os riscos trazidos a esses pacientes.

O Ministério da Saúde, pensando nesses pacientes, implantou o Programa Hiperdia, no qual se realiza o acompanhamento e tratamento de pa- 
cientes que são atendidos na rede ambulatorial do Sistema Único de Saúde (SUS) e que estão cadastrados como portadores de hipertensão arterial e/ou diabetes mellitus. Esse programa tem como objetivo combater amplamente esses agravos, estabelecendo metas e diretrizes para aumentar as ações de prevenção, diagnóstico, tratamento e controle dessas enfermidades, devendo haver uma reorganização do trabalho de atenção à saúde das unidades da rede básica dos serviços de saúde ${ }^{11}$. Mesmo diante dessas informações e sabendo da importância desse programa, podemos observar que ainda existe uma pequena parcela de profissionais que não participam do programa, respondendo de forma negativa ao esperado, pois 33,3\% dos entrevistados não fazem parte do Hiperdia.

No presente estudo foi investigada a associação estatística entre o conhecimento correto da definição de hipertensão arterial e algumas variáveis que determinam o perfil do profissional que trabalha nos ESFs, como: tempo que está no emprego; trabalhar em outro local; tempo de formado; ter pós-graduação; e participar do Hiperdia. Diante dos resultados obtidos, observou-se a associação estatística entre trabalhar em outro local e definição correta da HA. Esse dado pode se associar ao fato de os profissionais que têm mais de um ambiente de trabalho sentirem-se estimulados a buscar constante atualização a respeito de temas relevantes como esse, de modo que toda e qualquer forma de conhecimento é sempre interessante buscar, fazendo com que esse profissional se destaque mais.

Os resultados, aqui, obtidos mostraram que há algumas falhas no processo de atualização desses profissionais, pois falta um incentivo maior para que novas pesquisas sejam desenvolvidas com o mesmo perfil, de forma que cada vez mais seja trabalhado o tema, transmitindo mais conhecimento e havendo um interesse maior por parte dos cirurgiões-dentistas. Dessa forma, novos conhecimentos e pensamentos serão transmitidos, aprimorando, cada vez mais, a transmissão de saberes a respeito da hipertensão arterial, contribuindo não somente para o aprendizado do profissional, mas também para o bem-estar do usuário.

\section{Considerações finais}

- os profissionais que participaram da pesquisa mostraram ter uma percepção deficiente em relação à abordagem dos pacientes hipertensos;

- a maioria dos profissionais responderam, de forma incorreta, quanto à quantidade de anestésico que pode ser utilizado no atendimento a um paciente hipertenso compensado, além do não conhecimento por parte de mais da metade dos cirurgiões-dentistas em relação às interações medicamentosas entre os Aines e os anti-hipertensivos.

\section{Abstract}

Objective: to verify the knowledge of dentists of the Family Health Strategy (FHS) in the city of Patos, PB, Brazil, in serving users with the measurement of Arterial Hypertension (AH). Materials and methods: cross-sectional analytical study, with the application of a structured questionnaire to 21 professionals of the FHS units from the city of Patos, PB, Brazil. Data were analyzed using descriptive and inferential bivariate statistics (chi-square and Fischer's exact tests). Results: from the respondents, $85.7 \%$ could define arterial hypertension, but only $44.4 \%$ of them answered it correctly; $95.2 \%$ correctly answered the normal value of blood pressure. Xerostomia was the most mentioned change caused by the use of antihypertensive (85.71\%); Lidocaine was the anesthetic of choice $(61.9 \%)$; the most mentioned drug interaction (48.6\%) was of antihypertensive with non-steroidal anti-inflammatory, and of analgesics with acetylsalicylic acid (ASA). From the total respondents, $66.7 \%$ participated in the Hiperdia program, and $33.3 \%$ reported measuring blood pressure of users depending on the anamnesis performed. Statistical association was observed between working in another location and correctly determining $\mathrm{AH}(p=0.006)$. Conclusions: the professionals who participated in the survey showed relatively good knowledge with compatible behavior to what literature suggests, but still requiring further clarification on the subject, to meet their major difficulties.

Keywords: Local Anesthesia. Hypertension. Dentistry. Vasoconstrictors.

\section{Referências}

1. Oliveira AEM, Simone J L, Ribeiro, RA. Pacientes hipertensos e a anestesia na odontologia: devemos utilizar anestésicos locais associados ou não com vasoconstritores? HU Rev. 2010;36:69-75.

2. Ministério da Saúde (Brasil). Hipertensão arterial sistêmica para o Sistema Único de Saúde. Brasília: Ministério da Saúde; 2006a.

3. Salim MAA, Cançado RP, Carvalho BM, Zampirolli FA, Cabral AM, Moreira TG. Identificação da hipertensão arterial sistêmica e fatores de risco em pacientes atendidos nas clínicas de Cirurgia Bucomaxilofacial da Faculdade de Odontologia da Faesa (ES). Rev. bras. Odontol. 2011; 68:39-43.

4. Yagiela JA, Haymore TL. Management of the hypertensive dental patient. CDA J. 2007; 35:51-59.

5. Lima CLP, Rios PSS, Lima CM, Rios MC. Interações medicamentosas na hipertensão: papel do farmacêutico no acompanhamento clínico dos pacientes. Cadernos de Graduação - Ciências Biológicas e da Saúde 2011; 13:69-81.

6. Fortes ZB, Nigro D. Aspectos farmacológicos da interação anti hipertensivos e anti-inflamatórios não-esteroides. Revista Brasileira de Hipertensão 2005; 12:108-11.

7. Costa ANF, Vasconcelos RG, Vasconcelos MG, Queiroz LMG, Barboza CAG. Conduta Odontológica em Pacientes Hipertensos. Rev Bras Ci Saúde 2013; 17:287-92.

8. Nascimento EM, Santos MF, Martins VM, Cavalcanti AL, Menezes VA, Granville-Garcia AF. A bordagem odontológica de pacientes com hipertensão: um estudo de intervenção. RFO. 2011; 16:30-5. 
9. Frankfort-Nachimas C, Nachimias D. Research Methods in the social sciences. 4th ed. London: Edward Arnold; 1992.

10. Sociedade Brasileira de Hipertensão. Departamento de Hipertensão da Sociedade Brasileira de Cardiologia. Departamento de Hipertensão da Sociedade Brasileira de Nefrologia III diretrizes para uso da monitorização ambulatorial da pressão arterial. Rev Bras Hipertensão 2001; 8:143-53.

11. Ministério da Saúde (Brasil). Caderno de Atenção Básica Hipertensão Arterial; n. 15. 2006

12. Santos JC, Rumel D. Emergência medica na prática odontológica no Estado de Santa Catarina; ocorrência, equipamentos e drogas, conhecimento e treinamento dos cirurgiões-dentistas. Ciên Saúde Colet. 2006; 11:183-90.

13. Souza MOF, Perez ARHS, Souza TOF, Martins MAT, Bussadori SK, Fernandes KPS, et al. Incidência de alterações sistêmicas e uso de medicamentos em pacientes atendidos em clínica odontológica. Conscientiae Saúde 2007; 6:305-11.

14. Ribas TRC, Armonia PL. Avaliação crítica do comportamento dos clínicos em relação aos cuidados, à escolha e ao uso de anestésicos locais de emprego odontológico em pacientes hipertensos. Rev. Inst. Ciênc. Saúde 1997; 15:19-25.

15. Bronzo AL, Cardoso JRCG, Ortega KC, Mion JRD. Felypressin increases blood pressure during dental procedures in hypertensive patients. Arq Bras Cardiol. 2012; 99:724-31.

16. Little JW, Minn M. The impact on dentistry of recent advances in the management of hypertension. Oral Surg Oral Med Oral Pathol Oral Radiol Endod. 2005; 90:591-9.

17. Holm SW, Cunningham LLJR, Bensadoun E, Madsen MJ. Hypertension: classification, pathophysiology, and management during outpatient sedation and local anesthesia. J Oral Maxillofal Surg. 2006; 64:111-21.

18. Leslie AD, Luis JN. Manejo odontológico del paciente con hipertensión arterial. Rev Cient Cienc Salud Virtual. 2010; $2: 87-100$

19. Bavitz JB. Dental management of patients with hypertension. Dent Clin N Am. 2006; 50:547-62.

20. Indriago AJAA. Manejo odontologico del paciente hipertenso. Acta Odontol Venezoel 2007; 45:1-8

21. Cassolato SF, Turnbull RS. Xerostomia: clinical aspects and treatment. Gerodontology 2003; 20:64-77.

22. Pinto-Coelho CM, Silva-Souza YTC, Daré AMZ, Pereira ACCI, Cardoso CM. Implicações clínicas da xerostomia: abordagem sobre o diagnóstico e tratamento. Rev Assoc Paul Cir Dent. 2002; 56:295-300.

23. Santos MA, Montano TCP, Carvalho CMA, Neves ILI, Neves RS. Manifestações bucais decorrentes do uso de medicamentos anti-hipertensivos. Rev. Soc. Cardiol. 2007; 17:14-7.

24. Neves LS, Neves ILI, Giorgi DMA, Grupi CJ, César LAM, Hueb W. et al. Efeitos do uso da adrenalina na anestesia local odontológica em portador de coronariopatia. Arq Bras Cardiol. 2007; 88:545-51.

25. Claffey E, Reader AI, Nusstein J, Beck M, Weaver J. Anesthetic efficacy of articaine for inferior alveolar nerve blocks in patients with irreversible pulpitis. J Endod. 2004; 30:568-71.

26. Sá-Lima JR, Raldi FV, Gomes RM. O uso de anestésicos locais com vasoconstritores em pacientes cardiopatas. JBC. $2004 ; 8: 171-8$

27. Soares RG, Salles AA, Irala LED, Limongi OL. Como escolher um adequado anestésico local para as diferentes situações na clínica odontológica diária. Rev Sul-Bras Odontol. 2006; 3:35-40

28. Carvalho MHC, Colaço AL, Silva LBB, Salgado MCO. Aspectos farmacológicos dos inibidores da ECA e dos receptores de angiotensina II. Rev. Bras. Hipertens. 2005; 12:97102.
29. De Lucia R, Oliveira-Filho RM, Planeta CS, Callacci M, Avellar MCW. Farmacologia integrada. 3 ed. Rio de Janeiro: Livraria e Editora Revinter Ltda; 2007.

30. Secoli SR. Polifarmácia: Interações e reações adversas no uso de medicamentos por idosos. Rev. Bras. Enferm. 2010; 63(1):136-40.

\section{Endereço para correspondência:}

Tássia Cristina de Almeida Pinto-Sarmento

Rua Antônio de Sousa Lopes, no 100/apto 1802-C - Catolé

58410-180 Campina Grande-PB

Fones: (83) 33219231

(83) 88582771

E-mail: tassiapinto@yahoo.com.br

Recebido: 22/04/2014. Aceito: 14/12/2014. 